\title{
Discrete Wavelet transform and Chaotic Map based Watermark Hiding in Medical Images
}

\author{
Pratik Raj Baghel \\ M. Tech, \\ Digital Communication \\ Department of Electronics and Communication, \\ T.I.T. Advance, Anand nagar, Bhopal
}

\author{
Manish Gurjar \\ Head of Department, \\ Electronics and Communication, \\ T.I.T. Advance, Anand nagar, Bhopal
}

\begin{abstract}
Digital watermarking is technique to hide an invisible watermark in a host image. One such area of application could be medical image processing where watermark may contain sensitive information of the patient. Hence digital watermarking will help to secure patient data from unauthorized modifications. In this review paper, discrete wavelet transform (DWT) domain and chaotic system based medical image watermarking scheme has been proposed for hiding patient information in medical image to authenticate, in other words, to trace the origin of the image. Firstly Discrete Wavelet Transform has been applied on the host image, decomposing the image into four sub-bands namely LL, LH, $\mathrm{HL}$ and HH. Then LL band is chosen to hide the watermark. LL band is divided into $3 \times 3$ non overlapping blocks. Each bit of watermark is hidden into each block. To add more security, logistic map has been used through which chaotic watermark has been obtained, which has been embedded into the low frequency LL sub-band of medical image. The chaotic watermark has been embedded into each block by observing neighbor pixels conditions and modifying one of neighbor pixels. Experimental results of the proposed method have been compared with other existing medical image watermarking scheme. Experimental results were produced on three parameters namely Mean Square Error ( MSE ), Peak signal to noise ratio ( PSNR ) and Normalized Correlation ( $\mathrm{NC}$ ).
\end{abstract}

\section{General Terms}

DWT based watermark hiding for medical images.

\section{Keywords}

digital watermarking, Discrete Wavelet transform, Chaotic watermark, Logistic map, Mean Square Error ( MSE ), Peak Signal to Noise ratio ( PSNR ), Normalized correlation ( NC ).

\section{INTRODUCTION}

Digital image watermarking scheme is the procedure of embedding digital signature or information called watermark into the host image or digital image. [2] Copy right protection, monitoring, tracking of files are the application of watermarking. Watermarking techniques are divided into two categories visible and invisible. Invisible watermarking is fragile and robust instead of visible (logos and paper watermark). Fragile watermark is important to verify if protected files was tempered or not, and the robustness provides proof of ownership.[13] The scheme of watermarking is divided in to three phases embedding, attack (transmission) and extraction (detection). In the first phase, after embedding the binary watermark into the cover image with the secret key, a watermarked image is obtained. Second stage includes the transmission of watermarked image. Anybody tempering with the watermarked image, transmission error or noise with the watermarked image, these are called the attacks. And the last is extraction; this is an algorithm which reconstructs the watermark from the watermarked image. [13].

\section{TECHNIQUES OF WATERMARKING}

In the field of digital image watermarking there are several techniques. But generally it is categorized into two types namely Spatial domain and Transform or frequency domain watermarking technique.

In spatial domain, embedding the message in to the image pixels, this is the oldest called LSB method. In this method, we insert the watermark directly in to the Least Significant Bits of the pixels data [3] [6] [7]. The affect by LSB method low computational complexity and low PSNR occur. In the transfer domain, message embeds by slightly modifying the coefficients of the host image as controvert (opposed) to the pixel values. It makes some unobtrusive changes in the whole image. Comparing to the spatial methods it makes more robust to attack.

In the digital images there are number of transforms can be applied, but mainly some important and benificial transforms which rapidly used that are Discrete Fourier Transform (DFT), Discrete Cosine Transform (DCT) and Discrete Wavelet Transform(DWT). Fourier Transform is an operation that transforms a continuous function in to its frequency components. Similar transform for discrete valued function requires DFT. DCT is like DFT. It uses the real parts of the DFT coefficients. DCT has a property of "energy compaction". DCT provides higher frequency resolution but lower spatial resolution so that more frequency bands but difficult to recognize the spatial information. [15] At moderate bit rate DCT performs well, higher compression ratio but degrades thee quality of image, because of its artifacts resulting from the block based DCT. Solution of these problems introduces the wavelet Transform, it is based on small wave called wavelet. It decompose the original signal to the wavelet transform coefficients contains the position information. And reconstruct the original signal by IDWT[12] DWT is more efficient than the other transforms because of space and frequency localization with different resolution levels.[6] [8]

\section{WATERMARKING APPLICATION FOR MEDICAL PURPOSE}

There are several fields like telemedicine, banking, shopping, office work, broadcasting etc are going to be digital or have to been digitized, so in the digitalization the security of data or information also the main concern on it. There are several fields like telemedicine, banking, shopping, office work, broadcasting etc are going to be digital or have to been digitized, so in the digitalization the security of data or 
information also the main concern on it. Telemedicine is the field to support and provide health care through electronic information technologies when distance saprets the doctor and patients [14]

Digitalization of medical images and medical information facilitates over the internet huge amount of data storage and transmission, So, privacy protection of images or information has been an important issue in the management of patient's medical records. Manny hospitals and health care systems requires large amount of digital data storage and transmission, such as medical images, patient information, and administrative documents. Among these data, the patient information and medical images are very sensitive and need to be protected against any types of malicious attacks[14]. In the storage and transmission of digital medical images, Medical imaging system such as CT scan, MRI, X-ray require reliable security [9][10]

In medical image, the patients' information as a watermark has been embedded in to the medical image. As the doctor diagnose from medical images. To hide the information in the medical image requires the special care. Embeds the watermark without degrading the image quality [11][12]

In this paper, Chaotic watermark which obtained by the help of logistic map, and mainly DWT based image watermarking technique has been proposed. In the proposed watermarking scheme, patient information (as the binary image) has been embedded in to the corresponding medical image. Discrete wavelet transform has been applied to the cover or host medical image and by applied DWT, its decompose the image in to four sub-bands of low and high frequencies. The logistic map has been used to get the chaotic watermark from watermark image. Then, the chaotic watermark has been embedded in to frequency sub-band (LL) of medical image without degrading the image quality.

\section{RELATED TECHNOLOGIES}

\subsection{Discrete Wavelet Transform (DWT)}

Discrete Wavelet Transform is a mathematical tool for hierarchically decomposing an image. It is currently used in a wide variety of signal processing applications, such as in audio and video compression, removal of noise in audio, and the simulation of wireless antenna distribution.

Wavelet works on multi resolution theory and provides spatial and frequency description of an image. In terms of nonstationary signals wavelet transform also the very useful, Wavelets likes a small wave and the transform based on it of frequency variation and limited duration. The wavelet transform provides multi-resolution, Unlike the conventional Fourier Transform which is only applied on the stationary signals, temporal information is retained from this transformation. Wavelets are created by the mother wavelet which is fixed function. To analyse the signals at different scales and resolutions this is called multiresolution and this is the only fundamental idea behind the wavelet.

The image splits by DWT in to four sub-bands of low and high frequencies. Therefore, a digital image is decomposed in to one low (LL) and three high frequency sub-bands (HL, LH and $\mathrm{HH}$ ). The lowest frequency level (LL sub-band) lie in the approximation part of the image.

In low sub-band (LL), majority of the image energy concentrates the edge components of image are largely confined in the high frequency sub-bands. Three high frequency sub-bands consist of the LH,HL HH respectively vertical horizontal and diagonal detail components. It increases the robustness of watermarking scheme by embedding of watermark into low frequency sub-band.

But, as the most of energy concentrates in the low frequency sub-band, the embedding of watermark into this sub-band the image quality is degraded. Now if we embed watermark in high frequency sub-bands, human eye cannot detect because of less sensitivity of human eyes to changes in edges. But the drawback is that watermark embedding into the high frequency sub-bands decreases the robustness of watermarking scheme. In the proposed watermarking scheme, the watermark is embedded into the low frequency sub-band without degrading the image quality.[1]

\subsection{Logistic map}

In the area of Digital Image Watermarking, increasing the security level is our main concern. Now a days or from some years chaotic map is used to increase the security level in the image processing one of the simplest chaotic maps that is logistic map, for image encryption the logistic map provides the efficient and secure way.

There has most attractive and beneficial feature of logistic map in information hiding is its extreme sensitivity on initial conditions. The logistic map [1] is described by-

$$
\mathrm{X}(\mathrm{i}+1)=\mu^{*} \mathrm{x}(\mathrm{i}) *\{1-\mathrm{x}(\mathrm{i})\}
$$
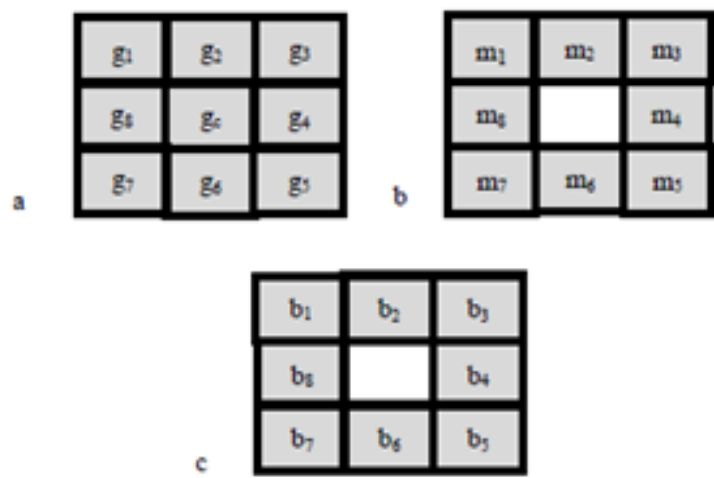

Fig 1: (a) gray values of $3 \times 3$ blocks (gi); (b) gray differences (mi) and (c) binary bits (bi) according to the sign of gray differences.

where, $0<\mu \leq 4$. The value of $\mu$ and $\mathrm{x}(1)$ are the initial conditions for logistic map. All the sequences generated by logistic map are highly sensitive to these initial conditions. Therefore, two random logistic sequences generated from different initial conditions are uncorrelated. In this paper, logistic map with initial conditions $\mu=3.90$ and $\mathrm{x}(1)=0.4545$ has been used to generate chaotic watermark. Fig.1 shows two different random configurations of logistic map.[1]

\section{PROPOSED METHOD}

In this section, the base paper method of embedding and extraction algorithm has been explained.

\subsection{Embedding Algorithm}

The patient information or the watermark image is embedded into the medical image as follows- 
1) Firstly, DWT is applied to the host or original medical image to decompose the image into low and high frequency sub-bands. After performing DWT on the image, non- overlapping sets- LL, HL, LH and HH can be obtained. Then, low frequency sub-band (LL) divided in to $3 \times 3$ non-overlapping blocks.

2) For each block, center pixel considered as the threshold and the pixels surrounding the center pixel are considered as the neighbor pixels. Fig. 1 depicts the $3 \times 3$ block representation.

3) The gray differences between centre pixel and neighbour pixels are calculated. The differences can be obtained by-

$\mathrm{mi}=\mathrm{gi}-\mathrm{gc}$,

where, $i=1$ to 8 . mi are the gray differences, gi are the gray values of neighbour pixels and gc is the gray value of the centre pixel

4) According to the sign of the gray differences, binary bits ( 1 and 0$)$ are assigned as follows-
$\mathrm{bi}=1$
; if $\mathrm{mi} \geq 0$
bi $=0$
; otherwise

5) Logical Exclusive OR (XOR) operation is applied to the bits those are obtained according to the sign of the gray differences. The XOR results can be obtained by-

$\mathrm{ex}=\mathrm{b} 1 \oplus \mathrm{b} 2 \oplus \mathrm{b} 3 \oplus \ldots \ldots \ldots \ldots \oplus \mathrm{b} 8$

where, $\oplus$ indicates the XOR operator and ex represents binary results 0 or 1 .

6) The patient information is taken as the binary watermark image. Then, the chaotic watermark is obtained by applying XOR operation between binary watermark image and generated logistic map.

7) Watermark bits are embedded into the blocks of LL subband, one by one. In other words, one bit of watermark bit wise embedded into one block.

8) The bits of chaotic watermark are embedded into each block of LL by observing the neighbour pixels conditions. Let $\mathrm{w}$ be one of bits of chaotic watermark. The XOR result (ex) is compared with the value of w. If the value of $w$ is same as the value of ex, the neighbor pixels of the block will be unchanged. If the value of $w$ is not same as the value of ex, one of the neighbour pixels will be modified to make the result of ex consistent with corresponding result of $\mathrm{w}$. This modification of one of neighbour pixels is done by using following steps-

i) At first, the minimum value (min) is calculated from the absolute gray differences (|mi|).

ii) From the neighbour pixels, the pixel (gmin) which gives minimum gray difference is detected. If minimum value is obtained from multiple neighbour pixels, any one of multiple pixels can be used.

iii) Gray value of this detected pixel is modified as follows-

$\mathrm{gm}=(\mathrm{gmin}-\min )-\beta \quad ;$ if $\mathrm{bmin}=1$

$\mathrm{gm}=(\mathrm{gmin}+\min )+\beta \quad ;$ if $\mathrm{bmin}=0$ where, gm is the modified gray value of pixel gmin, which gives minimum gray difference. bmin represents the value of binary bit according to the gray difference sign, which is obtained from the difference between gmin and gc. $\beta$ is the scaling factor.

9) Finally, the watermarked image can be obtained after performing inverse wavelet transform.

\subsection{Extraction Algorithm}

The watermark or the patient information is extracted from the watermarked medical image as follows-

1) DWT is applied to the watermarked image to obtain low frequency (LL) sub-band of watermarked image.

2) Low frequency sub-band (LL) is divided into $3 \times 3$ nonoverlapping blocks.

3) Gray differences between centre pixel and neighbour pixels of $3 \times 3$ blocks are calculated.

4) Binary bits ( 1 and 0 ) are assigned according to the sign of the gray differences. If the gray difference of a neighbour is negative, the binary bit of that neighbour is zero (0). On the other hand, if the gray difference of a neighbour is greater than or equal to zero, the binary bit of that neighbour is one (1). For 8 neighbour pixels, 8 binary bits can be obtained.

5) Logical Exclusive OR (XOR) operation is performed on the binary bits. The chaotic watermark bit can be extracted from the XOR result of the corresponding block.

6) Finally, the watermark image is obtained from the extracted chaotic watermark by applying XOR between logistic map and extracted chaotic watermark.

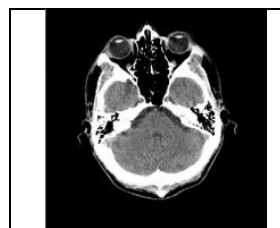

(a)

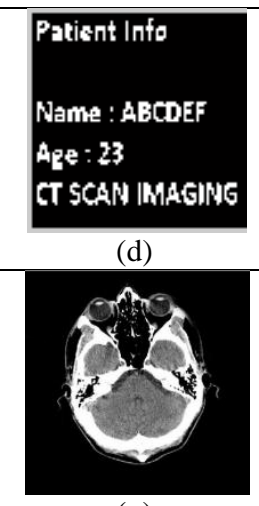

(g)

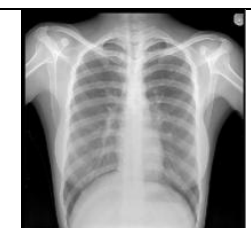

(b)

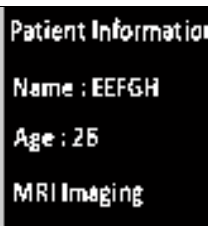

(e)

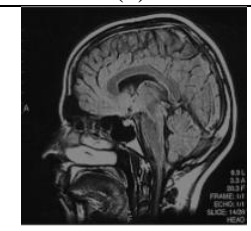

(h)

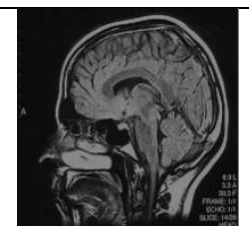

(c)

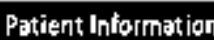

Nome : БHHJk

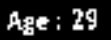

x.ray Imaging
Fig 2: (a)-(c) original medical images; (d)-(f) binary watermark images; (g)-(i) watermarked medical images and (j)-(l) corresponding extracted watermarks. 


\section{EXPERIMENTAL RESULTS}

Three parameters have been chosen to check for performance of proposed method. They are-
a) Mean square error ( MSE )
b) Peak to Signal noise ratio ( PSNR )
c) Normalised Correlation ( $\mathrm{NC}$ )

MSE and PSNR are used to measure the quality of embedded image while $\mathrm{NC}$ has been used to check the quality of embedded watermark. The formula to calculate these parameters are as under-

a) MSE:-

$M S E=\frac{1}{M N}\left[\sum_{x=0}^{M-1} \sum_{y=0}^{N-1}\left(I(x, y)-I^{\prime}(x, y)\right)^{2}\right]$

b) PSNR:-

$$
P S N R=10 \log _{10} \frac{\max ^{2}}{M S E}
$$

c) $\mathrm{NC}:-$

$$
N C=\frac{\sum_{x=0}^{m-1} \sum_{y=0}^{n-1}\left(W(x, y) \times W^{\prime}(x, y)\right)}{\sum_{x=1}^{m-1} \sum_{y=1}^{n-1} W^{\prime}(x, y)^{2}}
$$

Table 1.. Results of MSE and PSNR of proposed method

\begin{tabular}{|l|l|l|l|l|l|l|}
\hline & \multicolumn{2}{|l|}{ CT Scan } & \multicolumn{2}{l|}{ MRI Image } & \multicolumn{2}{l|}{ X-ray Image } \\
\hline $\begin{array}{l}\text { Scaling } \\
\text { Factor }\end{array}$ & MSE & PSNR & MSE & PSNR & MSE & PSNR \\
\hline 1 & 1.65 & 45.95 & 1.43 & 46.57 & 0.31 & 53.178 \\
\hline 1.5 & 1.72 & 45.79 & 1.51 & 46.35 & 0.35 & 52.691 \\
\hline 2 & 1.76 & 45.68 & 1.58 & 46.15 & 0.4 & 52.138 \\
\hline 2.5 & 1.81 & 45.55 & 1.68 & 45.88 & 0.46 & 51.549 \\
\hline 3 & 1.98 & 45.17 & 1.81 & 45.56 & 0.56 & 50.681 \\
\hline 3.5 & 2.05 & 45.01 & 1.9 & 45.34 & 0.61 & 50.285 \\
\hline 4 & 2.1 & 44.91 & 2 & 45.13 & 0.68 & 49.825 \\
\hline 4.5 & 2.16 & 44.78 & 2.12 & 44.87 & 0.76 & 49.327 \\
\hline 5 & 2.41 & 44.31 & 2.3 & 44.51 & 0.91 & 48.531 \\
\hline
\end{tabular}

Table 2. Performance of NC against attacks

\begin{tabular}{|l|l|l|l|}
\hline Attack Type & CT scan & MRI & x-ray \\
\hline $\begin{array}{l}\text { Inverse transform } \\
\text { attack }\end{array}$ & 0.98 & 0.95 & 0.93 \\
\hline Croping (10\%) & 0.95 & 0.92 & 0.90 \\
\hline Salt and pepper & 0.89 & 0.91 & 0.91 \\
\hline
\end{tabular}

Table 3. MSE and PSNR comparison with method-[10]

\begin{tabular}{|c|c|c|c|c|c|c|c|}
\hline \multirow[t]{2}{*}{ Image } & \multirow[t]{2}{*}{ Size } & \multicolumn{2}{|c|}{ Method-[10] } & \multicolumn{2}{|c|}{ Method-[11] } & \multicolumn{2}{|c|}{$\begin{array}{l}\text { Proposed } \\
\text { Method }\end{array}$} \\
\hline & & MSE & PSNR & MSE & PSNR & MSE & PSNR \\
\hline $\begin{array}{l}\text { CT } \\
\text { scan }\end{array}$ & $\begin{array}{l}510 \\
\times \\
510\end{array}$ & 2.11 & 44.7 & 1.79 & 45.6 & 1.65 & 45.9 \\
\hline MRI & $\begin{array}{l}510 \\
\times \\
510\end{array}$ & 2.22 & 44.7 & 1.66 & 45.9 & 1.55 & 46.2 \\
\hline$X-R a y$ & $\begin{array}{l}510 \\
\times \\
510\end{array}$ & 2.16 & 44.6 & 1.13 & 47.6 & 0.33 & 52.9 \\
\hline
\end{tabular}
and method-[11]

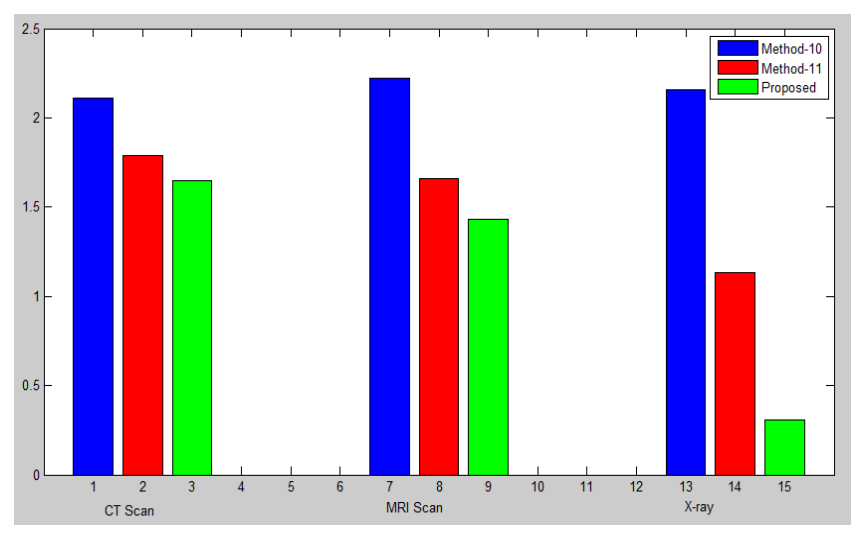

Fig 3: MSE comparison of proposed method with Method-[10] and Method-[11] 


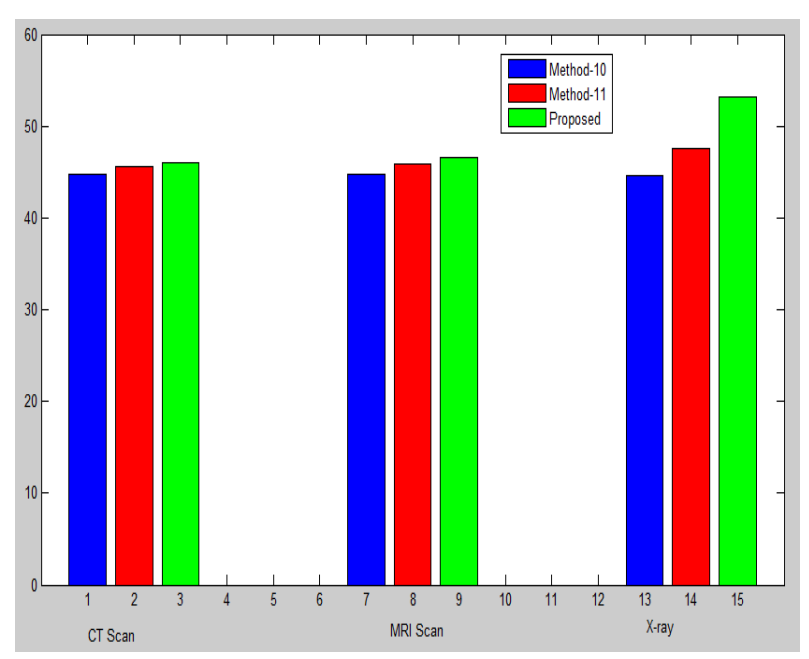

Fig 4: PSNR comparison of proposed method with Method-[10] and Method-[11]

Table 4. MSE and PSNR comparison with base method-[1] and proposed method

\begin{tabular}{|c|c|c|c|c|c|}
\hline \multirow[t]{2}{*}{ Image } & \multirow[t]{2}{*}{ Size } & \multicolumn{2}{|c|}{ Base Method[1] } & \multicolumn{2}{|c|}{$\begin{array}{l}\text { Proposed } \\
\text { Method }\end{array}$} \\
\hline & & $M S E$ & PSNR & $M S E$ & PSNR \\
\hline $\begin{array}{l}C T \\
\text { scan }\end{array}$ & $\begin{array}{l}510 \\
\times \\
510\end{array}$ & 1.63 & 46.01 & 1.65 & 45.93 \\
\hline$M R I$ & $\begin{array}{l}510 \\
\times \\
510\end{array}$ & 1.24 & 47.20 & 1.55 & 46.21 \\
\hline X-Ray & $\begin{array}{l}510 \\
\times \\
510\end{array}$ & 0.72 & 49.58 & 0.33 & 52.91 \\
\hline
\end{tabular}

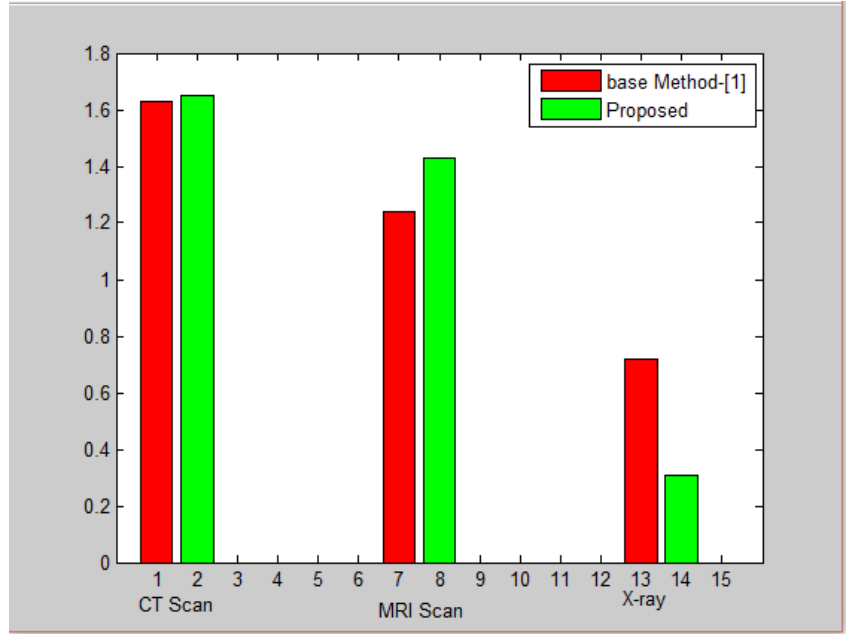

Fig 5: MSE comparison of proposed method with base method

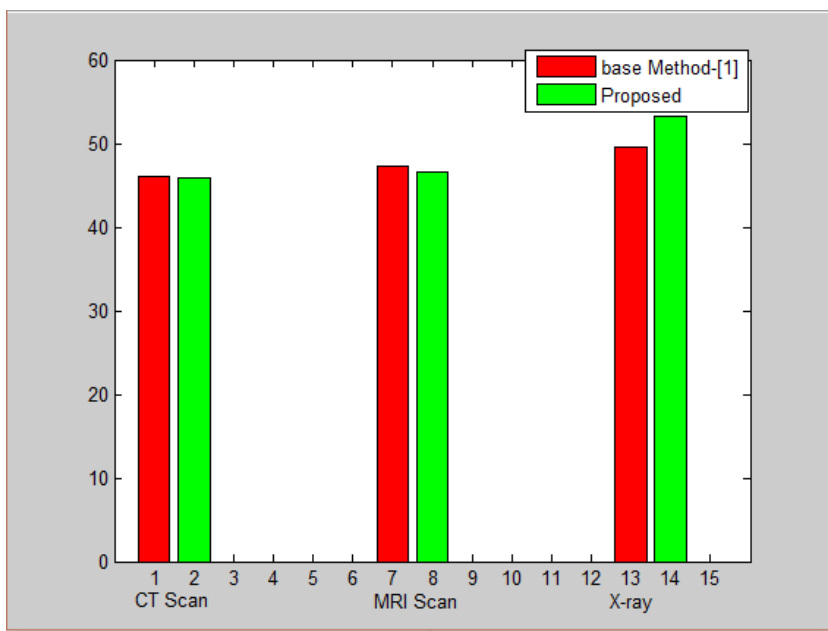

Fig 6: PSNR comparison of proposed method with base method

\section{CONCLUSION}

In this paper, we successfully reviewed the existing watermarking scheme against parameters like MSE, PSNR and NC. We have also concluded that inspite of hiding the watermark in the low frequency wavelet sub band of host image, which is most sensitive to human viewing. The results of the parameters could be varied on the different-different images. In this method our outcomes of CT scan and MRI has been little deviated but about to similar from the existing method but in X-ray image we could achieve very high PSNR and low MSE even compared with existing method. Further the embedded watermark was successfully hidden in the image and is not visible in the host image. Hence we conclude that proposed method effectively hides watermark images effectively outperforms the existing methods. In the future prospects scholars can perform another different attacks to different images for research also two level DWT can be perform.

\section{REFERENCES}

[1] Md. Moniruzzaman, Md. Abul Kayum Hawlader and Md. Foisal Hossain "Wavelet Based Watermarking Approach of Hiding Patient Information in Medical 
Image for Medical Image Authentication” Department of Electronics and Communication Engineering Khulna University of Engineering and Technology, (KUET) Khulna-9203, Bangladesh.. 978-1-4799-62884/14/\$31.00 @2014 IEEE

[2] Cox IJ, Matthew LM, Jeffrey AB, et al., "Digital Watermarking and Steganography," Second edition, Burlington, MA: Morgan Kaufmann Publishers (Elsevier), 2007

[3] Chandra M. B. and Srinivas K. S., "Robust Multiple Image Watermarking Scheme using Discrete Cosine Transform with Multiple Descriptions," International Journal of Computer Theory and Engineering, vol. 1, pp. 1793-8201, 2009.

[4] Imen Fourati Kallel, Mohamed Kallel, Mohamed Salim BOUHLEL, "A Secure fragile Watermarking Algorithm for medical Image Authentication in the DCT Domain," IEEE, 2006.

[5] V. Solachidis, I. Pitas, "Circularly symmetric watermark embedding in 2-D DFT domain," IEEE Trans. Image Process., vol. 10, pp. 1741-1753, 2001.

[6] B.L. Gunjal, R.R. Manthalkar, "Discrete Wavelet Transform Based Strongly Robust Watermarking Scheme for Information Hiding in Digital Images," Third Int. Conf. Emerging Trends in Engineering and Technology. India, pp. 124-129, November 2010.

[7] Fengmei LIANG, Lijia WANG, "An Improved WaveletBased Color Image Watermark Algorithm," Journal of Computational Information Systems 7, vol. 6, pp. 20132020, 2011

[8] S. A. Mostafa, N. El-sheimy, A. Tolba, F. Abdelkader, and H. M. Elhindy, "Wavelet packets-based blind watermarking for medical image management," The open biomedical engineering journal, vol. 4, 2010.
[9] Giakoumaki, Sotiris Pavlopoulos, and Dimitris Koutsouris, "Multiple Image Watermarking Applied to Health Information Management", IEEE Trans. on information technology in biomedicine, vol. 10, no. 4, October 2006.

[10] S. C. Rathi and V. S. Inamdar, "Medical Images Authentication Through Watermarking Preserving ROI," Health Informatics - An International Journal (HIIJ), vol.1, no.1, August 2012.

[11] A.Giakoumaki, S. Pavlopoulos, D. Koutsouris, "Multiple Digital Watermarking Applied to Medical Imaging," Proceedings of the 2005 IEEE, Engineering in Medicine and Biology 27th Annual Conference, Shanghai, China, September 1-4, 2005.

[12] Giakoumaki A, Pavlopoulos S and Koutsouris. "A multiple watermarking scheme applied to medical image management," in: Engineering in Medicine and Biology Society. IEMBS'04. 26th Annual International Conference of the IEEE, vol. 2, pp. 3241-3244, 2004.

[13] S. Rawat, B. Raman, "A chaotic system based fragile watermarking scheme for image tamper detection," Sci. Direct International Journal of Electronics and Communication, vol. 65, pp. 840-847, 2011.

[14] G. Coatrieux, L. Lecornu, "A Review of Image Watermarking Applications in Healthcare " Members, IEEE, Ch. Roux, Fellow, IEEE, B. Sankur, Member, IEEE

[15] Mohd Rizal Mohd Isa, Salem Aljareh "Biometric Image Protection Based on Discrete Cosine Transform Watermarking Technique" School of Engineering University of Portsmouth Portsmouth, United Kingdom, 978-1-4673-4810-2/12/\$31.00 O2012 IEEE. 